\title{
ERRATUM TO: A CONTRIBUTION TO THE SCIENTIFIC FOUNDATIONS OF SYSTEMS ENGINEERING: SOLUTION SPACES AND REQUIREMENTS
}

\author{
Alejandro Salado ${ }^{1}$ Roshanak Nilchiani $^{2}$ Dinesh Verma $^{2}$ \\ ${ }^{1}$ Grado Department of Industrial and Systems Engineering, Virginia Tech, Blacksburg VA, 24061, USA \\ asalado@vt.edu $(\square)$ \\ ${ }^{2}$ School of Systems and Enterprises, Stevens Institute of Technology, Hoboken NJ, 07030, USA \\ rnilchia@stevens.edu \\ dverma@stevens.edu
}

Erratum to: J Syst Sci Syst Eng

10.1007/s11518-016-5315-3

The article A Contribution to the Scientific Foundations of Systems Engineering: Solution Spaces and Requirements written by Alejandro Salado, Roshanak Nilchiani and Dinesh Verma, has been revised due to a missing part the authors forgot to add. The missing part goes at the end of Appendix B, Page 36, right after the sentence "The new compliant space is reduced to four systems."

The online version of the original article can be found under doi: $10.1007 / \mathrm{s} 11518-016-5315-3$ 
$r_{1}$ : The system shall have a mass lower than 5 $\mathrm{kg}$.

$$
\begin{aligned}
& R_{B}=R_{A} \cup\left\{r_{1}\right\} \\
& \operatorname{mass}\left(Z_{i}\right)=i k g, i=1, \cdots, 10 \\
& C S_{R_{B}}=\left\{Z_{1}, Z_{2}, Z_{3}, Z_{4}\right\} \\
& \# C S_{R_{B}}=4 .
\end{aligned}
$$

This compliant space is used as a reference to assess how inapplicable and other non-system requirements affect both its size and the compliance of the systems.

\section{$\underline{\text { Case } 1 \text { Inapplicable requirements }}$}

The inapplicable requirement $r_{2}$ is added to the set of requirements. Because the requirement is actually not referring to the system, but to a deliverable the company supplying the system has to provide, the compliant space remains the same, and thus so does its size.

$r_{2}$ : The supplier shall deliver a development plan.

$$
\begin{aligned}
& r_{2} \notin R_{B} \\
& R_{C}=R_{B} \cup\left\{r_{2}\right\} \\
& C S_{R_{C}}=\left\{Z_{1}, Z_{2}, Z_{3}, Z_{4}\right\}=C S_{R_{B}} \\
& \# C S_{R_{C}}=4=\# C S_{R_{B}} .
\end{aligned}
$$

In case the company does not deliver the required document, i.e., verification of such a requirement is false, the systems are still able to fulfill the initial set of requirements before the inapplicable requirement was added. Consequently, compliance of an inapplicable requirement does not affect compliance of the other requirements.

Case 2 Overlapping requirements as another type of non-system requirements

The overlapping requirement $r_{3}$ is added to the set of requirements. All systems in the compliant space are compliant to that requirement and, as a consequence, the compliant space remains identical and thus its size is unmodified.

$r_{3}:$ The system shall have a mass lower than 10 $\mathrm{kg}$.

$$
\begin{aligned}
& r_{3} \notin R_{B} \\
& R_{D}=R_{B} \cup\left\{r_{3}\right\} \\
& C S_{R_{D}}=\left\{Z_{1}, Z_{2}, Z_{3}, Z_{4}\right\}=C S_{R_{B}} \\
& \# C S_{R_{D}}=4=\# C S_{R_{B}} .
\end{aligned}
$$

However, if $r_{3}$ cannot be met $r_{1}$ would also not be met, thus affecting compliance of the system. In practical terms, that means that the development entity would not be able to find $Z_{1}$ through $Z_{10}$.

$$
c\left(Z_{i}, r_{3}\right)=F A L S E \rightarrow c\left(Z_{i}, r_{1}\right)=F A L S E, \forall i .
$$

$\underline{\text { Example 2 Definition of overlapping }}$ requirements

Consider the set of requirements formed by $r_{1}$ and $r_{2}$.

Dominant overlap:

$r_{1}$. Mass $<1 \mathrm{~kg}$ Case A. Actual system mass $=0.7 \mathrm{~kg}$.

$r_{2}$. Mass $<10 \mathrm{~kg} \quad$ Case B. Actual system mass $=7 \mathrm{~kg}$.

In Case A, both $r_{1}$ and $r_{2}$ are compliant. As can be seen, being compliant to $r_{1}$ automatically results in compliance to $r_{2}$. In Case $\mathrm{B}$, however, only $r_{2}$ is compliant. In this case, compliance to $r_{2}$ does not automatically results in compliance to $r_{1}$.

Mutual overlap:

$r_{1}$. Mass $<1 \mathrm{~kg}$

Case A. Actual system mass = $0.7 \mathrm{~kg}$. 
$r_{2}$. Weight on Earth $<1 \mathrm{~kg}$ Case B. Actual compliance to $r_{1}$ automatically results in system weight compliance to $r_{2}$. In case $\mathrm{B}$, both requirements on Earth $=0.5 \quad$ are also compliant because a weight of $0.5 \mathrm{~kg}$ on $\mathrm{kg}$. Earth without other external force requires a mass

In Case A, both requirements are compliant of $0.5 \mathrm{~kg}$. Therefore, compliance to $r_{2}$ because a mass of $0.7 \mathrm{~kg}$ automatically results in automatically results in compliance to $r_{1}$. a weight of $0.7 \mathrm{~kg}$ on Earth. Therefore, 